\title{
ON EXACT SOLUTIONS FOR DIVIDEND STRATEGIES \\ OF THRESHOLD AND LINEAR BARRIER TYPE \\ IN A SPARRE ANDERSEN MODEL*
}

\author{
BY \\ Hansjörg Albrecher, JÜrgen Hartinger \\ AND STEFAN THONHAUSER
}

\begin{abstract}
For the classical Cramér-Lundberg risk model, a dividend strategy of threshold type has recently been suggested in the literature. This strategy consists of paying out part of the premium income as dividends to shareholders whenever the free surplus is above a given threshold level. In contrast to the well-known horizontal barrier strategy, the threshold strategy can lead to a positive infinitehorizon survival probability, with reduced profit in terms of dividend payments. In this paper we extend several of these results to a Sparre Andersen model with generalized Erlang(n)-distributed interclaim times. Furthermore, we compare the performance of the threshold strategy to a linear dividend barrier model. In particular, (partial) integro-differential equations for the corresponding ruin probabilities and expected discounted dividend payments are provided for both models and explicitly solved for $n=2$ and exponentially distributed claim amounts. Finally, the explicit solutions are used to identify parameter sets for which one strategy outperforms the other and vice versa.
\end{abstract}

\section{KEYWORDS}

Sparre Andersen model; dividend payments, piece-wise deterministic Markov processes, ruin probability.

\section{INTRODUCTION}

In collective risk theory, the Sparre Andersen model to describe the surplus process of an insurance portfolio has a long history, starting with the original paper [30]. In that model, the claim counting process $\left(N_{t}\right)_{t \geq 0}$ for time $t$ is assumed to be an ordinary renewal process, which can be written as

$$
N_{t}=\min \left\{k: T_{1}+\ldots+T_{k+1}>t\right\}, t \geq 0,
$$

* Supported by the Austrian Science Fund Project P-18392. 
where $\left(T_{i}\right)_{i \in \mathbb{N}}$ is the sequence of independent interarrival times.

This renewal assumption allows for more flexibility than the classical CramérLundberg risk process (in which $N_{t}$ constitutes a homogeneous Poisson process) and enables to some extent contagion between claim occurrences (see for instance Rolski et al. [27] and Asmussen [5] for textbook treatments of these models). As usual, the premium inflow is assumed to be continuous over time with constant intensity $c$, and the individual claim amounts $\left(Y_{i}\right)_{i \in \mathbb{N}}$ are independent and identically distributed positive random variables with distribution function $F_{Y}$ and mean $\mu<\infty$. Then, for initial capital $u$, the risk reserve process $\left(R_{t}\right)_{t \geq 0}$ of the insurance portfolio at time $t$ is given by

$$
R_{t}=u+c t-S_{t} .
$$

where $S_{t}=\sum_{i=1}^{N_{t}} Y_{i}$ denotes the aggregate claim amount at time $t$. The net profit condition in this model is given by $c>\mu / \mathbb{E}\left(T_{i}\right)$. Typical quantities studied in this context are the time of ruin

$$
\tau(u)=\inf \left\{t>0 \mid R_{t}<0, R_{0}=u\right\},
$$

and the probability of ruin

$$
\psi(u)=P\left\{\tau(u)<\infty \mid R_{0}=u\right\} .
$$

Whereas the Poisson claim number process in the classical Cramér-Lundberg model implies that $\left(T_{i}\right)_{i \in \mathbb{N}}$ is a sequence of independent exponential random variables and consequently the interarrival times possess a lack-of-memory property, this Markovian property does not carry over to general renewal risk processes. However, the assumption that each $T_{i}$ follows a generalized Erlang $(n)$ distribution (i.e. each $T_{i}$ is a convolution of $n$ independent exponentially distributed random variables with parameters $\lambda_{1}, \ldots, \lambda_{n}$ ) allows to utilize some analytical tools that are usually restricted to Markov processes. This approach was recently exploited by Li \& Garrido [23] and Gerber \& Shiu [19] for the study of the discounted penalty function (which contains the ruin probability as well as other ruin-related quantities such as the distribution of the ruin deficit and the time of ruin) in such an $\operatorname{Erlang}(n)$ renewal risk model. In earlier papers, expressions for the probability of ultimate ruin and the Laplace transform of the time to ruin for the special case of an Erlang(2) risk process had been obtained by Dickson [10] and Dickson \& Hipp $[12,13]$ and for the same model Cheng \& Tang [7] had used the discounted penalty function to get integrodifferential equations for the moments of the surplus before ruin and the deficit at ruin.

The analytical tractability of this Erlangian renewal setup has also been used to include a horizontal dividend barrier strategy in the analysis, i.e. whenever the surplus reaches a certain level, all incoming premiums are immediately paid out as dividends. In this context, Li \& Garrido [24] derived properties of the resulting risk process (generalizing earlier results for the Cramér-Lundberg 
model with horizontal barrier in Lin et al. [26]) and Albrecher et al. [1] calculated moments of dividend payments resulting from this strategy (generalizing corresponding results for the Cramér-Lundberg model with horizontal barrier in Dickson \& Waters [14]). For a textbook treatment of horizontal barrier strategies, see for instance Dickson [11].

However, one considerable disadvantage of the horizontal barrier strategy is that the resulting surplus process will lead to ruin with probability 1 , which typically makes this strategy inappropriate in practice. More than that, even if one is just interested in maximizing expected discounted dividend payouts, the horizontal payout strategy is not optimal. While for the compound Poisson model there are some optimality properties for horizontal barriers (see e.g. Gerber [15], Azcue \& Muler [6] and Schmidli [28]), it can be shown that horizontal barrier strategies are not optimal in the Sparre Andersen model (see Albrecher \& Hartinger [2]). These two issues motivate to look at alternative dividend payment strategies that allow for a positive probability of survival and still have a satisfying level of dividend payouts. In particular, it is desirable to look for model assumptions that at the same time enable analytical expressions for decision quantities related to solvency and profit, so that the parameters of the strategy can be tuned towards a given target.

Among these, there is the so-called threshold dividend strategy, where one fixes a level $b>0$ and no dividends are paid out if the surplus level is below $b$. Whenever the surplus is above $b$, dividends are paid with intensity $a, 0<a<c$ (and the surplus increases with intensity $c-a$ ) until the surplus falls again below $b$ due to the occurrence of a claim. Finally, the dividend payments are stopped at the time of ruin. So the dynamics of the modified risk process $R^{t h r}$ are given by

$$
\begin{aligned}
& d R_{t}^{t h r}=c d t-d S_{t}, \quad 0 \leq R_{t}^{t h r}<b, \\
& d R_{t}^{t h r}=(c-a) d t-d S_{t}, R_{t}^{t h r} \geq b, \\
& d D_{t}^{t h r}=a d t, \quad R_{t}^{t h r} \geq b,
\end{aligned}
$$

where $\left(D_{t}^{t h r}\right)_{t \geq 0}$ denotes the accumulated dividend payments at time $t$ (note that $a=c$ corresponds to the horizontal barrier strategy). The expected discounted dividends of such a strategy are given by

$$
W(u, b)=\mathbb{E}\left(\int_{0}^{\tau(u)} a I_{\left\{R_{t}^{t h r} \geq b\right\}} e^{-\delta t} d t \mid R_{0}^{t h r}=u\right),
$$

where $\delta>0$ is the discounting factor (another interpretation for $\delta>0$ is that it reflects the preference of a shareholder to receive payments earlier rather than later according to an exponential utility function). Observe that $\psi(u)=1$ if the process above $b$ does not fulfill the net profit condition $(c-a) \mathbb{E}\left(T_{i}\right)>\mu$.

The threshold dividend strategy was discussed for the Cramér-Lundberg model in Asmussen [5] and recently studied in detail by Lin \& Pavlova [25] and Gerber \& Shiu [21]; for a diffusion setup, see also Gerber \& Shiu [20]). In this 
paper we will investigate the threshold dividend strategy for a renewal model with generalized Erlang(n) interarrival times.

The second strategy we will focus on is the so-called linear barrier dividend strategy, where the barrier $b_{t}=b+(c-a) t(b>0,0<a<c)$ grows linearly in time and dividends are paid out with intensity $a$ whenever the surplus reaches $b_{t}$, while the reserve increases with intensity $c-a$. On the other hand, nothing is paid out when the surplus is below the barrier. Dividend payments again stop at the event of ruin. The dynamics of the modified risk process $R^{\text {lin }}$ are thus given by

$$
\begin{aligned}
& d R_{t}^{\text {lin }}=c d t-d S_{t}, 0 \leq R_{t}^{\text {lin }}<b_{t}, \\
& d R_{t}^{\text {lin }}=(c-a) d t-d S_{t}, R_{t}^{\text {lin }}=b_{t}, \\
& d D_{t}^{\text {lin }}=a d t, \quad R_{t}^{\text {lin }}=b_{t} .
\end{aligned}
$$

The expected discounted dividend payments are in this case

$$
W(u, b)=\mathbb{E}\left(\int_{0}^{\tau(u)} a I_{\left\{R_{t}^{l i n} \geq b_{t}\right\}} e^{-\delta t} d t \mid R_{0}^{l i n}=u, b_{0}=b\right) .
$$

The linearity of the barrier enables the application of classical techniques developed for the horizontal barrier model for the computation of quantities of interest (such as martingale techniques (see e.g. Gerber [16])). Historically, together with the positivity of the corresponding survival probability, this was one of the reasons to consider linear barriers. Explicit formulae for $W(u, b)$ and $\psi(u, b)$ for light-tailed claim sizes were derived in Gerber [17] and Siegl \& Tichy [29]. Recently, the discounted penalty function and higher moments of discounted dividend payments for the linear barrier strategy were investigated in Albrecher et al. [3]. However, up to now all results in connection with linear barriers are available in the Cramér-Lundberg model only. In this paper we will generalize this analysis to Erlang renewal models.

Figure 1 depicts the two strategies for a sample path that leads to ruin in both cases.

An objection sometimes raised against the linear barrier model is the fact that the strategy depends on the point in time, i.e. the payment strategy is different for each $t$ for an otherwise identical situation. However, if one is forced to fix a dividend strategy at time 0 and is interested in both maximizing $W(u, b)$ and keeping the ruin probability $\psi(u, b)$ below a specified level, it is intuitively clear that for small $t$ one will try to deduct a high dividend amount possibly involving some higher risk, whereas for larger $t$ the main focus will be on securing the survival (due to the discount factor $\delta$, the dividend contributions at this later stage will only be marginal). Indeed, as will be illustrated in Section 4, for higher values of $\delta$ the linear barrier model often outperforms the threshold model in terms of finding a compromise between the values of $W(u, b)$ and $\psi(u, b)$. 

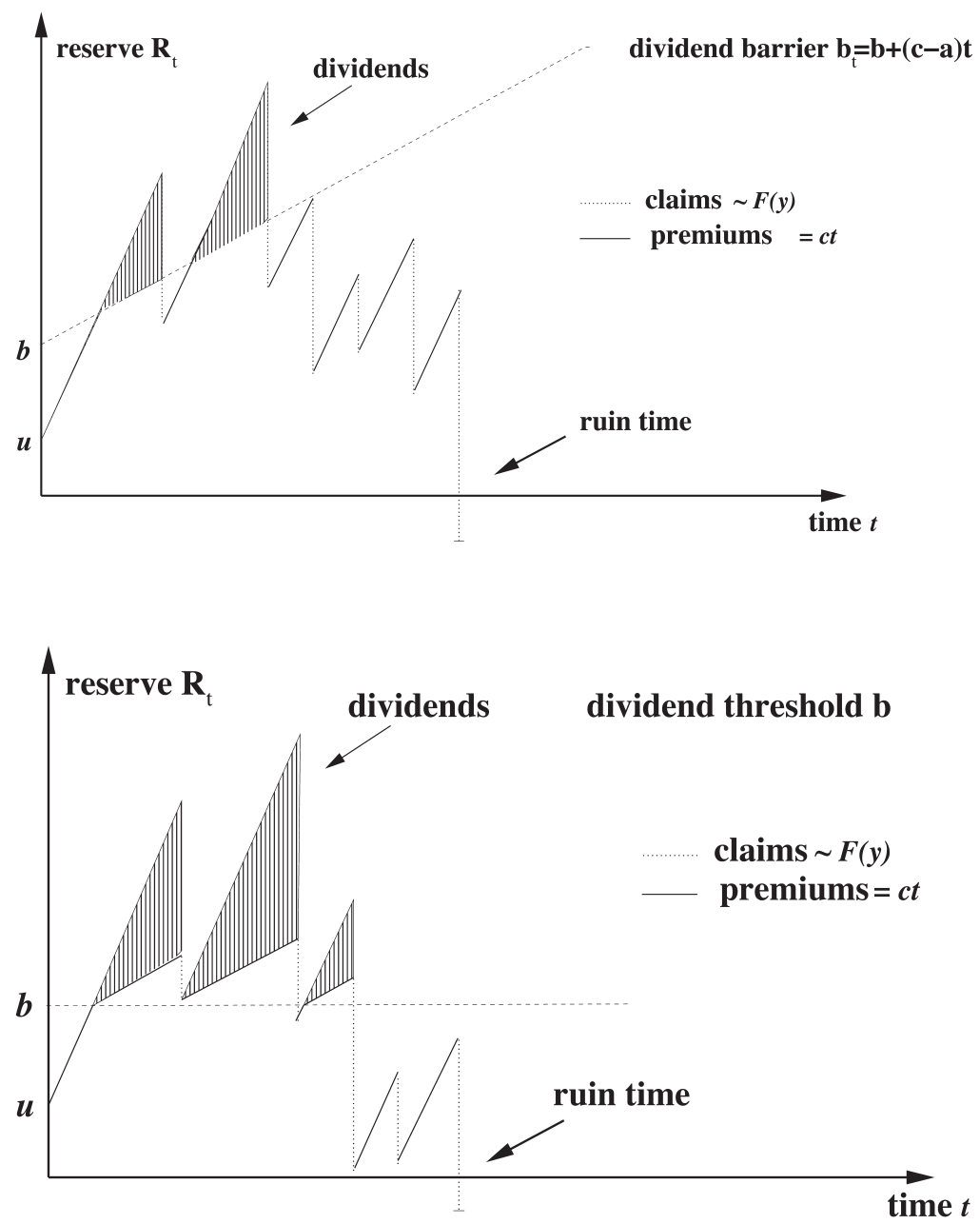

FIGURE 1: The linear barrier- and the threshold strategy for a sample path of $R_{t}$.

In this paper, we will derive (partial) integro-differential equations ((P)IDE's) for the ruin probability and moments of dividend payments for both the threshold and the linear barrier strategy in the Sparre Andersen model with generalized Erlang $(n)$ interclaim times. These equations can in principle be solved explicitly whenever the individual claim size distribution itself follows a generalized Erlang distribution. We will demonstrate this solution procedure for the case of Erlang(2) interclaim times and exponential (i.e. Erlang(1)) claim amounts. The explicit formulae pave the way for a fast numerical assessment of the performance of these dividend strategies for a given set of parameters. 
In Section 2, we use the differential approach to establish a PIDE for the moment-generating function of discounted dividend payments for the threshold strategy in the Sparre Andersen model with $\operatorname{Erlang}(n)$ interclaim times. Subsequently, the corresponding IDE's for arbitrary moments of the discounted dividend payments and for the ruin probability are derived. These equations are explicitly solved for $n=2$ and exponential claim amounts in Section 2.4. Section 3 provides PIDE's for the survival probability as well as the expected discounted dividend payments in the linear barrier model with $\operatorname{Erlang}(n)$ interclaim times. As an alternative to the differential approach, these are derived using the framework of piece-wise deterministic Markov processes. Again, an explicit solution is provided for $n=2$ and exponential claim amounts and the exact results are compared with simulation in Section 3.2.4. Finally, in Section 4 the analytical results of the previous sections are used to compare the performance of the threshold and linear barrier model for various sets of parameters. Section 5 concludes.

\section{The Threshold Dividend Strategy}

\subsection{A system of PIDEs for the moment-generating function}

Let us decompose every inter-occurrence time with generalized Erlang(n)-distribution into the independent sum of $n$ exponential random variables with possibly different parameters $\lambda_{1}, \ldots, \lambda_{n}$, each causing a "sub-claim" of size 0 and at the time of the $n$-th sub-claim an actual claim with distribution function $F_{Y}$ occurs. This is done by defining $n$ states for the risk process (see e.g. [1]). Starting at time 0 in state 1 , every sub-claim causes a transition to the next state and at the time of occurrence of the $n$-th sub-claim, an actual claim with distribution function $F_{Y}$ occurs and the risk process jumps into state 1 again. Let

$$
M^{(j)}(u, y, b)=\mathbb{E}\left(\exp \left(y \int_{0}^{\tau(u)} e^{-\delta t} a I_{\left\{R_{t}^{t h r}>b\right\}} \mathrm{d} t\right) \mid R_{0}^{t h r}=u, \text { state }=j\right)
$$

denote the moment-generating function of the discounted dividend payments, given that the risk process starts in state $j(j=1, \ldots, n)$.

Furthermore we split up the moment-generating functions in two regions below and above the barrier,

$$
M^{(j)}(u, y, b)=M_{1}^{(j)}(u, y, b) I_{\{u<b\}}+M_{2}^{(j)}(u, y, b) I_{\{u \geq b\}} \cdot
$$

For $j=1, \ldots, n-1$, we condition on the occurrence of a claim within an infinitesimal time interval, which gives

$$
\begin{aligned}
M_{1}^{(j)}(u, y, b) & =\left(1-\lambda_{j} d t\right) M_{1}^{(j)}\left(u+c d t, y e^{-\delta d t}, b\right) \\
& +\lambda_{j} d t M_{1}^{(j+1)}\left(u+c d t, y e^{-\delta d t}, b\right)+o(d t),
\end{aligned}
$$


and

$$
\begin{aligned}
M_{2}^{(j)}(u, y, b) & =\left(1-\lambda_{j} d t\right) e^{y a d t} M_{2}^{(j)}\left(u+(c-a) d t, y e^{-\delta d t}, b\right) \\
& +\lambda_{j} d t e^{y a d t} M_{2}^{(j+1)}\left(u+(c-a) d t, y e^{-\delta d t}, b\right)+o(d t) .
\end{aligned}
$$

The analogous equations for $j=n$ are

$$
\begin{aligned}
M_{1}^{(n)}(u, y, b)= & \left(1-\lambda_{n} d t\right) M_{1}^{(n)}\left(u+c d t, y e^{-\delta d t}, b\right) \\
& +\lambda_{n} d t \int_{0}^{u+c d t} M_{1}^{(1)}\left(u+c d t-z, y e^{-\delta d t}, b\right) \mathrm{d} F_{Y}(z) \\
& +\lambda_{n} d t \int_{u+c d t}^{\infty} \mathrm{d} F_{Y}(z)+o(d t), \\
M_{2}^{(n)}(u, y, b)= & \left(1-\lambda_{n} d t\right) e^{y a d t} M_{2}^{(n)}\left(u+(c-a) d t, y e^{-\delta d t}, b\right) \\
& +\lambda_{n} d t e^{y a d t} \int_{0}^{u+c d t} M^{(1)}\left(u+(c-a) d t-z, y e^{-\delta d t}, b\right) \mathrm{d} F_{Y}(z) \\
& +\lambda_{n} d t e^{y a d t} \int_{u+c d t}^{\infty} \mathrm{d} F_{Y}(z)+o(d t) .
\end{aligned}
$$

Taylor expansion and collection of suitable terms leads to the following partial (integro-)differential equations $(j=1, \ldots, n-1)$ :

$$
\begin{aligned}
& M_{1}^{(j+1)}(u, y, b)=\left(\frac{-c \frac{\partial}{\partial u}+\lambda_{j}+\delta y \frac{\partial \cdot}{\partial y}}{\lambda_{j}}\right) M_{1}^{(j)}(u, y, b), \\
& M_{2}^{(j+1)}(u, y, b)=\left(\frac{-(c-a) \frac{\partial \cdot}{\partial u}+\left(\lambda_{j}-y a\right)+\delta y \frac{\partial \cdot}{\partial y}}{\lambda_{j}}\right) M_{2}^{(j)}(u, y, b),
\end{aligned}
$$

and

$$
\begin{aligned}
& \left(\begin{array}{c}
-c \frac{\partial \cdot}{\partial u}+\lambda_{n}+\delta y \frac{\partial \cdot}{\partial y} \\
\lambda_{n}
\end{array}\right) \begin{array}{l}
M_{1}^{(n)}(u, y, b) \\
-\left(1-F_{Y}(u)\right)-\int_{0}^{u} M_{1}^{(1)}(u-z, y, b) \mathrm{d} F_{Y}(z)=0
\end{array} \\
& \left(\begin{array}{c}
\left.\frac{-(c-a) \frac{\partial \cdot}{\partial u}+\left(\lambda_{n}-y a\right)+\delta y \frac{\partial \cdot}{\partial y}}{\lambda_{n}}\right) \\
-\left(1-F_{Y}(u)\right)-\int_{0}^{u} M^{(1)}(u-z, y, b) \mathrm{d} F_{Y}(z)=0
\end{array}\right.
\end{aligned}
$$

The quantity of eventual interest is $M^{(1)}(u, y, b):=M(u, y, b)$, which from the above equations is seen to be the solution of the following system of partial integro-differential equations: 


$$
\begin{aligned}
0= & \left(\prod_{j=1}^{n} \frac{-c \frac{\partial \cdot}{\partial u}+\lambda_{j}+\delta y \frac{\partial}{\partial y}}{\lambda_{j}}\right) M_{1}(u, y, b)-\left(1-F_{Y}(u)\right) \\
& -\int_{0}^{u} M_{1}(u-z, y, b) \mathrm{d} F_{Y}(z), \\
0= & \left(\prod_{j=1}^{n} \frac{-(c-a) \frac{\partial \cdot}{\partial u}+\left(\lambda_{j}-y a\right)+\delta y \frac{\partial \cdot}{\partial y}}{\lambda_{j}}\right) M_{2}(u, y, b)-\left(1-F_{Y}(u)\right) \\
& -\int_{0}^{u} M(u-z, y, b) \mathrm{d} F_{Y}(z)
\end{aligned}
$$

(note that the product $y \frac{\partial}{\partial y}$ in the above operator is not commutative). Boundary conditions are given by

$$
\begin{aligned}
& \lim _{b \rightarrow \infty} M_{1}(u, y, b)=1, \\
& \lim _{u \rightarrow \infty} M_{2}(u, y, b)=e^{y a / \delta} .
\end{aligned}
$$

Moreover, at $u=b$, by continuity we have to have

$$
\lim _{u \rightarrow b^{+}} M_{2}^{(j)}(u, y, b)=\lim _{u \rightarrow b^{-}} M_{1}^{(j)}(u, y, b)
$$

for all states $j=1, \ldots, n$, which translates into

$$
\left.\left((c-a) \frac{\partial^{+} \cdot}{\partial u}-\delta y \frac{\partial^{+} \cdot}{\partial y}+y a\right)^{j-1} M_{2}\right|_{u=b}=\left.\left(c \frac{\partial^{-} \cdot}{\partial u}-\delta y \frac{\partial^{-}}{\partial y}\right)^{j-1} M_{1}\right|_{u=b},
$$

where the derivatives are assumed to be one-sided.

\subsection{The moments of the discounted dividends}

The results of the previous subsection can be used to derive an integro-differential equation for the $m$ th moment $W_{m}(u, b)$ of the discounted sum of dividend payments $(m \in \mathbb{N})$. Again, we write

$$
W_{m}(u, b)=W_{m, 1}(u, b) I_{\{u<b\}}+W_{m, 2}(u, b) I_{\{u \geq b\}} .
$$

For $\delta>0$, the quantity $W_{m}(u, b)$ is bounded for every $m \in \mathbb{N}$. Hence, with the representation

$$
M(u, y, b)=1+\sum_{m=1}^{\infty} \frac{y^{m}}{m !} W_{m}(u, b),
$$


and the equations (1)-(3), a comparison of coefficients of $y^{m}$ gives

$$
\begin{gathered}
\left(\prod_{j=1}^{n} \frac{-c \frac{\partial \cdot}{\partial u}+\lambda_{j}+\delta \bar{\Delta}}{\lambda_{j}}\right) W_{m, 1}(u, y, b)-\int_{0}^{u} W_{m, 1}(u-z, y, b) \mathrm{d} F_{Y}(z)=0, \\
\left(\prod_{j=1}^{n} \frac{-(c-a) \frac{\partial \cdot}{\partial u}+\left(\lambda_{j}-a \Delta\right)+\delta \bar{\Delta}}{\lambda_{j}}\right) W_{m, 2}(u, y, b)-\int_{0}^{u} W_{m}(u-z, y, b) \mathrm{d} F_{Y}(z)=0,
\end{gathered}
$$

with the operators $\Delta W_{m}:=m W_{m-1}, \bar{\Delta} W_{m}:=m W_{m}$. Moreover, $W_{0}=1, W_{-i}=0$ $(i \in \mathbb{N})$. Here, the product $\Delta \bar{\Delta}$ of operators is not commutative and is given by $(\Delta \bar{\Delta}) W_{m}=\bar{\Delta}\left(\Delta W_{m}\right)=m(m-1) W_{m-1}$ and $(\bar{\Delta} \Delta) W_{m}=\Delta\left(\bar{\Delta} W_{m}\right)=m^{2} W_{m-1}$.

We have the boundary conditions:

$$
\begin{aligned}
& \lim _{b \rightarrow \infty} W_{m, 1}(u, y, b)=0, \\
& \lim _{u \rightarrow \infty} W_{m, 2}(u, y, b)=\left(\frac{a}{\delta}\right)^{m} .
\end{aligned}
$$

Moreover, by assuming that all moments are continuous we have

$$
\left.\left(c \frac{\partial^{-} \cdot}{\partial u}\right)^{j-1} W_{m, 1}\right|_{u=b}=\left.\left((c-a) \frac{\partial^{+}}{\partial u}+a \Delta\right)^{j-1} W_{m, 2}\right|_{u=b},(j=1, \ldots, n) .
$$

Remark 2.2. Note that the above formulas generalize equations (5.1)-(5.3) of Gerber \& Shiu [21], who studied the case $m=1, n=1$.

\subsection{Probability of ruin}

The probability of ruin is defined through

$$
\psi(u, b)=\mathbb{E}\left(I_{\{\tau<\infty\}} \mid R_{0}^{t h r}=u\right) .
$$

Let us again split the function in two regions below and above the barrier $b$,

$$
\psi(u, b)=\psi_{1}(u, b) I_{\{u<b\}}+\psi_{2}(u, b) I_{\{u \geq b\}} .
$$

Analogously to Section 2.1, one can now decompose the process into $n$ states and subsequently apply the differential approach to obtain

$$
\left(\prod_{j=1}^{n} \frac{\lambda_{j}-c \frac{\partial \cdot}{\partial u}}{\lambda_{j}}\right) \psi_{1}(u, b)-\left(1-F_{Y}(u)\right)-\int_{0}^{u} \psi_{1}(u-z, b) \mathrm{d} F_{Y}(z)=0,
$$




$$
\left(\prod_{j=1}^{n} \frac{\lambda_{j}-(c-a) \frac{\partial \dot{\partial}}{\partial u}}{\lambda_{j}}\right) \psi_{2}(u, b)-\left(1-F_{Y}(u)\right)-\int_{0}^{u} \psi(u-z, b) \mathrm{d} F_{Y}(z)=0 .
$$

The natural boundary conditions are

$$
\lim _{u \rightarrow \infty} \psi_{2}(u, b)=0
$$

and

$$
\lim _{b \rightarrow \infty} \psi_{1}(u, b)=\bar{\psi}(u)
$$

where $\bar{\psi}(u)$ denotes the ruin probability without dividend payments. Moreover, from the continuity assumptions,

$$
\left.\left((c-a) \frac{\partial^{+}}{\partial u}\right)^{j-1} \psi_{2}(u, b)\right|_{u=b}=\left.\lim _{u \rightarrow b^{-}}\left(c \frac{\partial^{-}}{\partial u}\right)^{j-1} \psi_{1}(u, b)\right|_{u=b},(j=1, \ldots, n) .
$$

Remark 2.2. For $n=1$ (i.e. the Cramér-Lundberg model), (6) and (7) appear implicitly in Asmussen [5] and Lin \& Pavlova [25].

\subsection{Erlang(2) interarrivals and exponential claims}

In principle, the above equations can be explicitly solved for arbitrary Erlang distributed claim sizes. In the following we will illustrate the solution procedure for the specific case of $\operatorname{Erlang}(2, \lambda)$ distributed interclaim times (i.e. $\lambda_{1}=$ $\lambda_{2}=\lambda$ and $\left.n=2\right)$ and $\operatorname{Exp}(\alpha)$ distributed claim amounts. From Section 2.2, we then obtain

$$
\left(\lambda+\delta m-c \frac{\partial \cdot}{\partial u}\right)^{2} W_{m, 1}-\lambda^{2} \int_{0}^{u} W_{m, 1}(u-z, b) \mathrm{d} F_{Y}(z)=0
$$

and

$$
\begin{aligned}
& \left(\lambda+\delta m-(c-a) \frac{\partial \cdot}{\partial u}\right)^{2} W_{m, 2}-\lambda^{2} \int_{0}^{u} W_{m}(u-z, b) \mathrm{d} F_{Y}(z) \\
& =a m\left(-2(c-a) \frac{\partial \cdot}{\partial u}+2 \lambda+(2 m-1) \delta\right) W_{m-1,2}-a^{2}(m-1) m W_{m-2,2} .
\end{aligned}
$$

together with the boundary conditions (4),(5),

$$
W_{m, 1}(b, b)=W_{m, 2}(b, b)
$$


and

$$
\left.c \frac{\partial W_{m, 1}}{\partial u}\right|_{u=b}=\left.(c-a) \frac{\partial W_{n, 2}}{\partial u}\right|_{u=b}+a m W_{m-1,2}(b, b) .
$$

Let us consider the case $m=1$. We then have

$$
\begin{gathered}
c^{2} W_{1,1}^{\prime \prime}(u, b)-2 c(\delta+\lambda) W_{1,1}^{\prime}(u, b)+(\delta+\lambda)^{2} W_{1,1}(u, b) \\
-\lambda^{2} \alpha e^{-\alpha u} \int_{0}^{u} W_{1,1}(v, b) e^{\alpha v} d v=0
\end{gathered}
$$

and

$$
\begin{gathered}
(c-a)^{2} W_{1,2}^{\prime \prime}(u, b)-2(c-a)(\delta+\lambda) W_{1,2}^{\prime}(u, b)+(\delta+\lambda)^{2} W_{1,2}(u, b)-a(2 \lambda+\delta) \\
-\lambda^{2} \alpha e^{-\alpha u} \int_{0}^{u} W_{1}(v, b) e^{\alpha v} d v=0
\end{gathered}
$$

together with

$$
W_{1,1}(b-, b)=W_{1,2}(b+, b),
$$

and

$$
\left.c \frac{\partial^{-} W_{1,1}}{\partial u}(u, b)\right|_{u=b}=(c-a) \frac{\partial^{+} W_{1,2}}{\partial u}(u, b)+\left.a\right|_{u=b} .
$$

Applying the operator $\left(\frac{\partial}{\partial u}+\alpha\right)$ to (11) and (12) yields the differential equations

$$
\begin{aligned}
0= & c^{2} W_{1,1}^{\prime \prime \prime}(u, b)+\left(\alpha c^{2}-2 c(\delta+\lambda)\right) W_{1,1}^{\prime \prime}(u, b)+\left((\lambda+\delta)^{2}-2 c \alpha(\delta+\lambda)\right) W_{1,1}^{\prime}(u, b) \\
& +\left(\alpha(\delta+\lambda)^{2}-\alpha \lambda^{2}\right) W_{1,1}(u, b), \\
0= & (c-a)^{2} W_{1,2}^{\prime \prime \prime}(u, b)+\left(\alpha(c-a)^{2}-2(c-a)(\delta+\lambda)\right) W_{1,2}^{\prime \prime}(u, b)+\left((\delta+\lambda)^{2}\right. \\
& -2 \alpha(c-a)(\delta+\lambda)) W_{1,2}^{\prime}(u, b)+\left(\alpha(\delta+\lambda)^{2}-\alpha \lambda^{2}\right) W_{1,2}(u, b)-a \alpha(2 \lambda+\delta)
\end{aligned}
$$

The solution of (15) is of the form

$$
W_{1,1}(u, b)=\sum_{i=1}^{3} A_{1}^{(i)}(b) e^{R_{1}^{(i)} u}
$$

where $R_{1}^{(1)}, R_{1}^{(2)}>0$ and $R_{1}^{(3)}<0$ are the three roots of

$$
(\delta+\lambda-c R)^{2}(R+\alpha)-\alpha \lambda^{2}=0,
$$

and $A_{1}^{(i)}(b)(i=1,2,3)$ are coefficients (depending on the value of $\left.b\right)$ to be determined in the following. Substitution in (11) then gives the condition 


$$
\sum_{i=1}^{3} \frac{A_{1}^{(i)}(b)}{R_{1}^{(i)}+\eta}=0
$$

On the other hand, equation (16) has a solution of the form

$$
W_{1,2}(u, b)=\frac{a}{\delta}+A_{2}^{(1)}(b) e^{R_{2}^{(1)} u},
$$

where $\frac{a}{\delta}$ is a particular solution of (16) and $R_{2}^{(1)}$ is the negative root of

$$
(\delta+\lambda-(c-a) R)^{2}(R+\alpha)-\alpha \lambda^{2}=0
$$

(that this equation has indeed exactly one negative root follows by a Rouchétype argument, see e.g. Gerber \& Shiu [19]). The coefficients of the positive roots have to be zero according to (4), hence these terms do not appear in (18).

Substituting (17) and (18) in (12), a comparison of coefficients gives

$$
\frac{A_{2}^{(1)} e^{R_{2}^{(1)} b}}{R_{2}^{(1)}+\alpha}-\frac{A_{1}^{(1)} e^{R_{1}^{(1)} b}}{R_{1}^{(1)}+\alpha}-\frac{A_{1}^{(2)} e^{R_{1}^{(2)} b}}{R_{1}^{(2)}+\alpha}-\frac{A_{1}^{(3)} e^{R_{1}^{(3)} b}}{R_{1}^{(3)}+\alpha}+\frac{a}{\alpha \delta}=0
$$

(note that the integral on the right hand side of (12) has to be written as $\left.\int_{0}^{u} W_{1}(v, b) e^{\alpha v} d v=\int_{0}^{b} W_{1,1}(v, b) e^{\alpha v} d v+\int_{b}^{u} W_{1,2}(v, b) e^{\alpha v} d v\right)$. Together with conditions (13) and (14), we hence obtain the explicit solution (17) and (18), where the coefficients are determined by the system of linear equations

$$
\left(\begin{array}{cccc}
0 & \frac{1}{R_{1}^{(1)}+\alpha} & \frac{1}{R_{1}^{(2)}+\alpha} & \frac{1}{R_{1}^{(3)}+\alpha} \\
-\frac{\alpha}{R_{2}^{(1)}+\alpha} e^{R_{2}^{(1)} b} & \frac{\alpha}{R_{1}^{(1)}+\alpha} e^{R_{1}^{(1)} b} & \frac{\alpha}{R_{1}^{(2)}+\alpha} e^{R_{1}^{(2)} b} & \frac{\alpha}{R_{1}^{(3)}+\alpha} e^{R_{1}^{(3)} b} \\
-e^{R_{2}^{(1)} b} & e^{R_{1}^{(1)} b} & e^{R_{1}^{(2)} b} & e^{R_{1}^{(3)} b} \\
-(c-a) R_{2}^{(1)} e^{R_{2}^{(1)} b} & c R_{1}^{(1)} e^{R_{1}^{(1)} b} & c R_{1}^{(2)} e^{R_{1}^{(2)} b} & c R_{1}^{(3)} e^{R_{1}^{(3)} b}
\end{array}\right)\left(\begin{array}{c}
A_{2}^{(1)} \\
A_{1}^{(1)} \\
A_{1}^{(2)} \\
A_{1}^{(3)}
\end{array}\right)=\left(\begin{array}{c}
0 \\
\frac{a}{\delta} \\
\frac{a}{\delta} \\
a
\end{array}\right) .
$$

For the ruin probability, one has to apply the operator $\left(\frac{\partial}{\partial u}+\alpha\right)$ on (6) and (7) to obtain the differential equations

$$
0=\left(\left(\frac{\partial}{\partial u}+\alpha\right)\left(\prod_{j=1}^{2} \frac{-c \frac{\partial \cdot}{\partial u}+\lambda_{j}}{\lambda_{j}}\right)-\alpha\right) \psi_{1}(u, b),
$$

and

$$
0=\left(\left(\frac{\partial \cdot}{\partial u}+\alpha\right)\left(\prod_{j=1}^{2} \frac{-(c-a) \frac{\partial \cdot}{\partial u}+\lambda_{j}}{\lambda_{j}}\right)-\alpha\right) \psi_{2}(u, b) .
$$


Thus for $i=1,2$, we have solutions of the form

$$
\psi_{i}(u, b)=A_{1}^{(3)}+\sum_{j=1}^{2} A_{i}^{(j)} e^{R_{i}^{(j)} u} .
$$

Consider the corresponding Lundberg equations

$$
0=(R+\alpha)\left(\prod_{j=1}^{2} \frac{-c R+\lambda_{j}}{\lambda_{j}}\right)-\alpha
$$

and

$$
0=(R+\alpha)\left(\prod_{j=1}^{2} \frac{-(c-a) R+\lambda_{j}}{\lambda_{j}}\right)-\alpha .
$$

For each of the two, one solution is 0 and both equations have exactly one negative solution (see again [19]), which are denoted by $R_{i}^{(1)}, i=1,2$. The remaining positive solutions are called $R_{i}^{(2)}$. Thus for $i=1,2$, one has

$$
\psi_{i}(u, b)=A_{i}^{(3)}+\sum_{j=1}^{2} A_{i}^{(j)} e^{R_{i}^{(j)} u},
$$

where the $A_{i}^{(j)}(b)$ depend on the choice of $b$. Condition (9) gives

$$
\lim _{b \rightarrow \infty} \psi_{1}(u, b)=\frac{\alpha+R_{1}^{(1)}}{\alpha} e^{R_{1}^{(1)} u}
$$

(cf. [19]) and (8) translates into

$$
\psi_{2}(u, b)=A_{2}^{(1)} e^{R_{2}^{(1)} u} .
$$

Thus, we now have four unknown constants. Two equations are obtained by using (10) for $j=1,2$ and the remaining two are found by a comparison of coefficients of the solutions in the IDE's (6) and (7). Altogether, the resulting system of equations is given by

$$
\left(\begin{array}{cccc}
e^{R_{1}^{(1)} b} & e^{R_{1}^{(2)} b} & e^{R_{1}^{(3)} b} & -e^{R_{2}^{(1)} b} \\
c R_{1}^{(1)} e^{R_{1}^{(1)} b} & c R_{1}^{(2)} e^{R_{1}^{(2)} b} & c R_{1}^{(3)} e^{R_{1}^{(3)} b} & -(c-a) R_{2}^{(1)} e^{R_{2}^{(1)} b} \\
\frac{\alpha}{\alpha+R_{1}^{(1)}} & \frac{\alpha}{\alpha+R_{1}^{(2)}} & \frac{\alpha}{\alpha+R_{1}^{(3)}} & 0 \\
\frac{1}{\alpha+R_{1}^{(1)}} e^{R_{1}^{(1)} b} & \frac{1}{\alpha+R_{1}^{(2)}} e^{R_{1}^{(2)} b} & \frac{1}{\alpha+R_{1}^{(3)}} e^{R_{1}^{(3)} b} & -\frac{1}{\alpha+R_{2}^{(1)}} e^{R_{2}^{(1)} b}
\end{array}\right)\left(\begin{array}{l}
A_{1}^{(1)} \\
A_{1}^{(2)} \\
A_{1}^{(3)} \\
A_{2}^{(1)}
\end{array}\right)=\left(\begin{array}{l}
0 \\
0 \\
1 \\
0
\end{array}\right) .
$$




\section{The Linear Dividend Barrier Strategy}

Consider a linear barrier of the form $b_{t}=b+(c-a) t$. With this strategy, dividends are paid out with intensity $a$ whenever $R_{t}^{\text {lin }}$ reaches $b_{t}$, while the reserve increases with intensity $c-a$ until the next claim occurs. On the other hand, nothing is paid if the surplus is below the barrier.

\subsection{Integro-differential equation for $U(u, b)$}

For convenience, let us think of the risk process with linear dividend barrier as a piecewise deterministic Markov process (PDMP) with $n$ external states (see e.g. Davis [9] or Rolski et al. [27]), where the transition from state $i$ to state $i+1$ is generated by an $\operatorname{Exp}\left(\lambda_{i}\right)$ random variable. This can again be interpreted as a decomposition of the interclaim time $T_{i}$ into $n$ exponentially distributed summands, see [1]. For $i=1, \ldots, n-1$ the process only changes the state, for $i=n$ the state moves to state 1 and a claim with distribution function $F_{Y}$ occurs. The generator $\mathbf{A}$ for a suitable function $g$ (depending on the state $i$, the risk process and the barrier $b$ ) is given by

$$
\begin{aligned}
c \frac{\partial g^{(i)}}{\partial u}(u, b) & +(c-a) \frac{\partial g^{(i)}}{\partial b}(u, b) \\
& +\lambda_{i}\left(g^{(i+1)}(u, b)-g^{(i)}(u, b)\right), \quad(0 \leq u<b, i=1, \ldots, n-1), \\
c \frac{\partial g^{(n)}}{\partial u}(u, b) & +(c-a) \frac{\partial g^{(n)}}{\partial b}(u, b) \\
& +\lambda_{n}\left(\int_{0}^{u} g^{(1)}(u-y, b) d F_{Y}(y)-g^{(n)}(u, b)\right), \quad(0 \leq u<b) .
\end{aligned}
$$

and at the barrier

$(c-a)\left(\frac{\partial g^{(i)}}{\partial u}(u, b)+\frac{\partial g^{(i)}}{\partial b}(u, b)\right)+\lambda_{i}\left(g^{(i+1)}(u, b)-g^{(i)}(u, b)\right),(u=b, i=1, \ldots, n-1)$,
$(c-a)\left(\frac{\partial g^{(n)}}{\partial u}(u, b)+\frac{\partial g^{(i)}}{\partial b}(u, b)\right)+\lambda_{n}\left(\int_{0}^{u} g^{(1)}(u-y, b) d F_{Y}(y)-g^{(n)}(u, b)\right),(u=b)$.

To get candidates for the survival probability $U^{(i)}(u):=1-\psi^{(i)}(u)$ (for initial state $i$ ) we have to solve the equations $\mathbf{A} U^{(i)}(u, b)=0$ together with the boundary conditions given below. From the equations above we get the following integro-differential equation for the survival probability in state 1 (which is the one we are in fact interested in):

$$
\prod_{i=1}^{n}\left(\lambda_{i}-c \frac{\partial \cdot}{\partial u}-(c-a) \frac{\partial \cdot}{\partial b}\right) U^{(1)}(u, b)=\prod_{i=1}^{n} \lambda_{i} \int_{0}^{u} U^{(1)}(u-y, b) d F_{Y} .
$$


By continuity at $u=b$, the boundary conditions are

$$
\left.\prod_{j=1}^{i-1}\left(\frac{\lambda_{j}-c \frac{\partial \cdot}{\partial u}-(c-a) \frac{\partial \cdot}{\partial b}}{\lambda_{j}}\right) \frac{\partial U^{(1)}}{\partial u}(u, b)\right|_{u=b}=0, \quad i=1, \ldots, n,
$$

where $\prod_{j=1}^{0} \cdot=1$. Moreover,

$$
\lim _{b \rightarrow \infty} U^{(1)}(u, b)=\bar{U}(u)
$$

(with $\bar{U}(u)$ denoting the survival probability in the renewal model without dividend payments) and $\lim _{u, b \rightarrow \infty} U^{(1)}(u, b)=1$ if $u$ and $b$ go to infinity uniformly (cf. [17]).

\subsubsection{Erlang(2) interarrivals and exponential claims}

In this section we look for an explicit solution for the survival probability in case of Erlang $(2, \lambda)$-distributed interclaim times and $\operatorname{Exp}(\alpha)$ claim amounts. Write $U(u, b):=U^{(1)}(u, b)$. The integro-differential equation then reads

$$
\left(\lambda-c \frac{\partial \cdot}{\partial u}-(c-a) \frac{\partial \cdot}{\partial b}\right)^{2} U(u, b)=\lambda^{2} \int_{0}^{u} U(u-y, b) \alpha e^{-\alpha y} d y
$$

together with the boundary conditions

$$
\begin{gathered}
\left.\frac{\partial U}{\partial u}(u, b)\right|_{u=b}=0 \\
\left.c \frac{\partial^{2} U}{\partial u^{2}}(u, b)\right|_{u=b}+\left.(c-a) \frac{\partial^{2} U}{\partial u \partial b}(u, b)\right|_{u=b}=0 \\
\lim _{b \rightarrow \infty} U(u, b)=\bar{U}(u), \\
\lim _{u, b \rightarrow \infty} U(u, b)=1 .
\end{gathered}
$$

Analogous to Section 2, equation (24) can be transformed into a partial differential equation with constant coefficients:

$$
\begin{aligned}
& \left(\lambda-c \frac{\partial \cdot}{\partial u}-(c-a) \frac{\partial \cdot}{\partial b}\right)^{2} \frac{\partial U}{\partial u}(u, b) \\
& \quad+\alpha\left(\lambda-c \frac{\partial \cdot}{\partial u}-(c-a) \frac{\partial \cdot}{\partial b}\right)^{2} U(u, b)-\alpha \lambda^{2} U(u, b)=0
\end{aligned}
$$


From (21) we have

$$
\bar{U}(u)=1-\frac{\alpha+R_{1,1}^{(1)}}{\alpha} e^{R_{1,1}^{(1)} u},
$$

where $R_{1,1}^{(1)}$ is the unique negative solution of (19).

We will construct an explicit solution to the above problem. Using fixedpoint arguments it follows that there is a unique solution to the problem, so we have actually solved the problem completely (see Albrecher \& Kainhofer [4] for details on the uniqueness argument in a related model and Cohen \& Down [8] in a queueing framework). The solution of (29) will be of the form

$$
\sum_{k=0}^{\infty} e^{S^{(k)} b}\left(A_{1}^{(k)} e^{R_{1}^{(k)} u}+A_{2}^{(k)} e^{R_{2}^{(k)} u}+A_{3}^{(k)} e^{R_{3}^{(k)} u}\right),
$$

where for each $k \geq 0$ the pairs $\left(S^{(k)}, R_{j}^{(k)}\right), j=1,2,3$, are zeroes of the polynomial

$$
P(R, S)=(R+\alpha)(\lambda-c R-(c-a) S)^{2}-\alpha \lambda^{2} .
$$

In the spirit of [29], the initial step $k=0$ is chosen in order to satisfy (27), i.e.

$$
S^{(0)}=0, R_{1}^{(0)}=0, A_{1}^{(0)}=1, A_{2}^{(0)}=-\frac{R_{1,1}^{(1)}+\alpha}{\alpha}, A_{3}^{(0)}=0 .
$$

If $S^{(k)}<0$ and all $S^{(k)}+R_{j}^{(k)}<0$ for all $k \geq 1, j=1,2,3$, then the two conditions (27) and (28) are fulfilled. Thus, if for some $k$ and $j, S^{(k)}+R_{j}^{(k)} \geq 0$, then necessarily $A_{j}^{(k)}=0$. It turns out that one can choose $A_{3}^{(k)}=0$ for all $k \geq 0$. Plugging (30) into the original equation (23), one obtains for each $k$

$$
A_{2}^{(k)}=-\frac{R_{2}^{(k)}+\alpha}{R_{1}^{(k)}+\alpha} A_{1}^{(k)} .
$$

Boundary condition (25) reduces to

$$
\sum_{k=0}^{\infty} A_{1}^{(k)}\left(R_{1}^{(k)} e^{\left(S^{(k)}+R_{1}^{(k)}\right) b}-\frac{R_{2}^{(k)}+\alpha}{R_{1}^{(k)}+\alpha} R_{2}^{(k)} e^{\left(S^{(k)}+R_{2}^{(k)}\right) b}\right)=0
$$

and condition (26) can be rewritten as

$$
\begin{aligned}
\sum_{k=0}^{\infty} A_{1}^{(k)} & \left(R_{1}^{(k)}\left(c R_{1}^{(k)}+(c-a) S^{(k)}\right) e^{\left(S^{(k)}+R_{1}^{(k)}\right) b}\right. \\
- & \left.\frac{R_{2}^{(k)}+\alpha}{R_{1}^{(k)}+\alpha} R_{2}^{(k)}\left(c R_{2}^{(k)}+(c-a) S^{(k)}\right) e^{\left(S^{(k)}+R_{2}^{(k)}\right) b}\right)=0 .
\end{aligned}
$$


For each $k$, the summand in (30) solves the integro-differential equation (29). For $k=0, S^{(0)}=R_{1}^{(0)}=0$, so only the second term produces an error concerning the two boundary conditions above (note that in Gerber [17] and Siegl \& Tichy [29], only one boundary condition had to be satisfied this way). For each of the two conditions, the first summand of a larger index $k$ in (30) will be used to correct for it. However, the second summand will again provide a mismatch with respect to this boundary condition and will itself be corrected by a first summand of higher index etc. It will turn out that these correction terms converge to zero and thus in the limit we have found the exact solution. In fact, the convergence is fast and with only a few terms of the series (30) the approximation to the exact value is satisfying.

\section{The deletion algorithm}

So, for a general step $k$, fix two new steps $\hat{k}>k$ and $k^{\prime}>k$ such that

$$
S^{(k)}+R_{2}^{(k)}=S^{\left(k^{\prime}\right)}+R_{1}^{\left(k^{\prime}\right)}=S^{(\hat{k})}+R_{1}^{(\hat{k})} .
$$

The coefficients $A_{1}^{\left(k^{\prime}\right)}$ and $A_{1}^{(\hat{k})}$ of the new steps have to solve the linear equations

$$
\begin{gathered}
\left(\frac{R_{2}^{(k)}+\alpha}{R_{1}^{(k)}+\alpha}\right) A_{1}^{(k)} R_{2}^{(k)}=A_{1}^{\left(k^{\prime}\right)} R_{1}^{\left(k^{\prime}\right)}+A_{1}^{(\hat{k})} R_{1}^{(\hat{k})}, \\
\left(\frac{R_{2}^{(k)}+\alpha}{R_{1}^{(k)}+\alpha}\right) A_{1}^{(k)} R_{2}^{(k)}\left(c R_{2}^{(k)}+(c-a) S^{(k)}\right)= \\
A_{1}^{\left(k^{\prime}\right)} R_{1}^{\left(k^{\prime}\right)}\left(c R_{1}^{\left(k^{\prime}\right)}+(c-a) S^{\left(k^{\prime}\right)}\right)+A_{1}^{(\hat{k})} R_{1}^{(\hat{k})}\left(c R_{1}^{(\hat{k})}+(c-a) S^{(\hat{k})}\right) .
\end{gathered}
$$

To that end, it is essential that $S^{\left(k^{\prime}\right)} \neq S^{(\hat{k})}$ and $R_{1}^{\left(k^{\prime}\right)} \neq R_{1}^{(\hat{k})}$. In the following it is shown that it is always possible to find two distinct roots $R_{1}^{\left(k^{\prime}\right)}$ and $R_{1}^{(\hat{k})}$ such that $S^{\left(k^{\prime}\right)}+R_{1}^{\left(k^{\prime}\right)}<0$ and $S^{(\hat{k})}+R_{1}^{(\hat{k})}<0$ and at the same time $S^{\left(k^{\prime}\right)}+R_{2}^{\left(k^{\prime}\right)}, S^{(\hat{k})}+$ $R_{2}^{(\hat{k})}<0$ holds.

For each fixed $S<0, P(R, S)$ has three real roots in $R$ which satisfy

$$
r_{1}(S)<0<r_{2}(S)<\frac{\lambda-(c-a) S}{c}<r_{3}(S)
$$

so that

$$
r_{2}(S)+S<\frac{\lambda}{c}+\frac{a}{c} S
$$

whence $r_{2}(S)+S<0$ if

$$
S<-\frac{\lambda}{a} .
$$


Later on we will see from the construction of $S^{(k)}$ that this is guaranteed. Moreover, it turns out that $\lim _{S \rightarrow \infty} r_{1}(S)=-\alpha$, whereas $r_{2}(S)$ and $r_{3}(S)$ do not have a finite limit.

On the other hand, if we fix $R$, then $P(R, S)=0$ has two solutions in $S$ given by

$$
s_{1,2}(R)=\frac{(c-a)(\alpha+R)(\lambda-c R) \pm \sqrt{\alpha(c-a)^{2} \lambda^{2}(\alpha+R)}}{(c-a)^{2}(\alpha+R)} .
$$

We get that $s_{2}(R)<0$ for $R \in\left(-\infty, r_{1}(0)\right) \cup\left(\min \left\{\frac{\lambda}{c}, r_{2}(0)\right\}, \min \left\{\frac{\lambda}{c}, r_{3}(0)\right\}\right) \cup$ $\left(\frac{\lambda}{c}, \infty\right)$. On the other hand, $s_{1}(R)>0$ if $R \in\left(\max \left\{\frac{\lambda}{c}, r_{1}(0)\right\}, \max \left\{\frac{\lambda}{c}, r_{2}(0)\right\}\right) \cup$ $\left(\max \left\{\frac{\lambda}{c}, r_{3}(0)\right\}, \infty\right)$.

Let us now turn to the determination of $R_{1}^{\left(k^{\prime}\right)}$ and $R_{1}^{(\hat{k})}$ for a given step $k$ so as to match (31). For that purpose, consider the polynomial

$$
P\left(R, S^{(k)}+R_{2}^{(k)}-R\right),
$$

which has three real roots $\left\{\tilde{R}_{1}, \tilde{R}_{2}, \tilde{R}_{3}\right\}$ in $R$. A closer look at its behavior reveals that we again have $\tilde{R}_{1}, \tilde{R}_{2}>0$ and $\tilde{R}_{3}<0$.

In the following it is shown that $S^{(k)}+R_{2}^{(k)}<0$, therefore the following choice is possible:

$$
\begin{array}{ll}
R_{1}^{\left(k^{\prime}\right)}:=\tilde{R}_{1}, & S^{\left(k^{\prime}\right)}=S^{(k)}+R_{2}^{(k)}-R_{1}^{\left(k^{\prime}\right)}, \\
R_{1}^{(\hat{k})}:=\tilde{R}_{2}, & S^{(\hat{k})}=S^{(k)}+R_{2}^{(k)}-R_{1}^{(\hat{k})} .
\end{array}
$$

If $S^{(k)}+R_{2}^{(k)}<0$, then clearly both $S^{\left(k^{\prime}\right)}+R_{2}^{\left(k^{\prime}\right)}<0$ and $S^{(\hat{k})}+R_{2}^{(\hat{k})}<0$. Moreover, from $R_{1}^{\left(k^{\prime}\right)}, R_{1}^{(\hat{k})}>0$ it follows that $S^{\left(k^{\prime}\right)}, S^{(\hat{k})}<0$, as required. Consequently, due to (33) it is always possible to choose $R_{2}^{\left(k^{\prime}\right)}, R_{2}^{(\hat{k})}<0$ as the negative solutions of $P\left(R, S^{\left(k^{\prime}\right)}\right)=0$.

Summarizing, starting with $S^{(0)}=0$, choose $R_{2}^{(0)}$ as the negative zero of $P(R, 0)$, so that

$$
S^{(0)}+R_{2}^{(0)}<0
$$

Then, the coefficients of two next steps $k^{\prime}$ and $\hat{k}$ are chosen according to (34) and (32). Subsequently, the same procedure is applied to each of the two steps and so on. By the above considerations and induction, $S^{(k)}+R_{2}^{(k)}<0$ holds for all $k \geq 0$. In addition,

$$
R_{2}^{\left(k^{\prime}\right)}+S^{\left(k^{\prime}\right)}=R_{2}^{(k)}+S^{(k)}-R_{1}^{\left(k^{\prime}\right)}+R_{2}^{\left(k^{\prime}\right)}<R_{2}^{(k)}+S^{(k)},
$$

since $R_{1}^{\left(k^{\prime}\right)}>0$ and $R_{2}^{\left(k^{\prime}\right)}<0$. So this sum decreases in every step of the algorithm and, moreover, $S^{\left(k^{\prime}\right)}<S^{(k)}$ (the same argument holds with $k^{\prime}$ replaced by $\hat{k}$ ).

A numerical illustration of this solution algorithm will be given in Section 3.2.4. 


\subsection{Integro-Differential equation for $W(u, b)$}

Since one of the boundary conditions to the equation for $W(u, b)$ will involve the expected discounted dividends of the linear barrier strategy in case the payments are continued after the event of ruin, we will first discuss this variant of the model.

\subsubsection{Dividend payments continue after ruin}

It is well-known that in this case it suffices to look at the process $z_{t}=b_{t}-R_{t}^{\text {lin }}$ (see e.g. [17]). Dividends are then paid whenever $z_{t}=0$. The resulting expected discounted dividends (with discounting factor $\delta>0$ ) are

$$
\begin{aligned}
V(z) & =\mathbb{E}\left(\int_{0}^{\infty} a I_{\left(z_{t}=0\right)} e^{-\delta t} d t \mid z_{0}=z\right), \\
\lim _{z \rightarrow \infty} V(z) & =0,
\end{aligned}
$$

which are bounded by

$$
\int_{0}^{\infty} a e^{-\delta t} d t=a / \delta
$$

As in Section 3.1 we think of $z=\left(z_{t}\right)_{t \geq 0}$ as a PDMP with $n$ external states. The generator $\mathbf{A}$ for a suitable function $g$ depending on the state $i$ and the process $z$ is given by

$$
\begin{aligned}
& \mathbf{A} g^{(i)}(z)=-a \frac{\partial g^{(i)}}{\partial z}(z)+\lambda_{i}\left(g^{(i+1)}(z)-g^{(i)}(z)\right),(z>0, i=1, \ldots, n-1), \\
& \mathbf{A} g^{(n)}(z)=-a \frac{\partial g^{(n)}}{\partial z}(z)+\lambda_{n}\left(\int_{0}^{\infty} g^{(1)}(z+y) d F_{Y}(y)-g^{(n)}(z)\right),(z>0),
\end{aligned}
$$

and at the boundary $z=0$ we get for the generator

$$
\lambda_{n}\left(\int_{0}^{\infty} g^{(1)}(y) d F_{Y}(y)-g^{(n)}(0)\right) \text { and } \lambda_{i}\left(g^{(i+1)}(0)-g^{(i)}(0)\right), \quad(i=1, \ldots, n-1
$$

Here, a function $g$ is suitable, if for all states $i$ it is absolutely continuous on $(0, \infty)$ and

$$
\mathbb{E}\left(\sum_{j, \sigma_{j}<t}\left|g^{(i)}\left(z_{\sigma_{j}}\right)-g^{(i)}\left(z_{\sigma_{j-}}\right)\right|\right)<\infty \quad \forall t>0,
$$

where $\left\{\sigma_{i}\right\}_{i \geq 1}$ denote the claim occurrence times (this condition is certainly fulfilled if $g$ is bounded). From Rolski et al. [27, Thm. 11.2.3] we know that for a suitable function $f$ which fulfills 


$$
\mathbf{A} f(z)-\delta f(z)+\gamma(z)=0
$$

the relation

$$
f\left(z_{0}\right)=\mathbb{E}\left(\int_{0}^{t_{0}} \gamma\left(z_{t}\right) e^{-\delta t} d t+e^{-\delta t_{0}} f_{\text {ter }}\left(z_{t_{0}}\right)\right)
$$

holds for any $t_{0}>0$ (and for a bounded function $f_{\text {ter }}$ the second summand vanishes for $\left.t_{0} \rightarrow \infty\right)$.

Let $V^{(i)}(z)$ denote the value of the expected dividends for initial state $i$ and set $\gamma(z)=a I_{(z=0)}$. Then we can write

$$
V^{(i)}\left(z, t_{0}\right)=\mathbb{E}\left(\int_{0}^{t_{0}} \gamma\left(z_{t}\right) e^{-\delta t} d t+e^{-\delta t_{0}} V_{t e r}\left(i, z_{t_{0}}\right)\right) .
$$

From the upper bound (35) it is clear that $V_{\text {ter }}$ is bounded and hence neglible in the limit $t_{0} \rightarrow \infty$ in the above expression. Hence $V^{(i)}(z)=V^{(i)}(z, \infty)$ is given as the solution of

$-a \frac{\partial V^{(i)}}{\partial z}(z)+\lambda_{i}\left(V^{(i+1)}(z)-V^{(i)}(z)\right)-\delta V^{(i)}(z)=0,(i=1, \ldots, n-1, z>0)$

and

$-a \frac{\partial V^{(n)}}{\partial z}(z)+\lambda_{n}\left(\int_{0}^{\infty} V^{(1)}(z+y) d F_{Y}(y)-V^{(n)}(z)\right)-\delta V^{(n)}(z)=0,(z>0)$.

For $z=0$ we get the boundary conditions

$$
\begin{aligned}
& \lambda_{i}\left(V^{(i+1)}(0)-V^{(i)}(0)\right)-\delta V^{(i)}(0)+a=0, i=1, \ldots, n-1, \\
& \lambda_{n}\left(\int_{0}^{\infty} V^{(1)}(y) d F_{Y}(y)-V^{(n)}(0)\right)-\delta V^{(n)}(0)+a=0 .
\end{aligned}
$$

Moreover, continuity of $V^{(i)}(z)$ implies $\frac{\partial V^{(i)}}{\partial z}(0)=-1$ for $i=1, \ldots, n$. Eventually, we are interested in the quantity $V(z)=V^{(1)}(z)$. From (36) we have

$$
V^{(i+1)}(z)=\prod_{j=1}^{i}\left(\frac{\delta+\lambda_{j}+a \frac{\partial \cdot}{\partial z}}{\lambda_{j}}\right) V^{(1)}(z)
$$

and together with (37) we arrive at

$$
\prod_{i=1}^{n} \lambda_{i} \int_{0}^{\infty} V^{(1)}(z+y) d F_{Y}(y)=\prod_{i=1}^{n}\left(\delta+\lambda_{i}+a \frac{\partial \cdot}{\partial z}\right) V^{(1)}(z), \quad(z>0)
$$


and for the boundary $z=0$

$$
\prod_{j=1}^{i-1}\left(\frac{\delta+\lambda_{j}+a \frac{\partial \cdot}{\partial z}}{\lambda_{j}}\right) V^{(1)}(0)=-1, \quad(i=1, \ldots, n) .
$$

\subsubsection{Dividend payments stop at ruin}

If the dividend payments stop at the event of ruin, the expected discounted dividend payments are given by

$$
W(u, b)=\mathbb{E}\left(\int_{0}^{\tau(u)} a I_{\left(R_{t}^{l i n}=b_{t}\right)} e^{-\delta t} d t \mid R_{0}^{l i n}=u, b_{0}=b\right) .
$$

The PDMP approach analogous to Section 3.2.1 leads to the PIDE

$$
\prod_{i=1}^{n} \lambda_{i} \int_{0}^{u} W(u-v, b) d F_{Y}(v)=\prod_{i=1}^{n}\left(\left(\delta+\lambda_{i}\right)-c \frac{\partial \cdot}{\partial u}-(c-a) \frac{\partial \cdot}{\partial b}\right) W(u, b),
$$

and boundary conditions are

$$
\begin{aligned}
V(z) & =\lim _{u \rightarrow \infty} W(u, u+z), \\
1 & =\left.\prod_{k=1}^{j-1}\left(\frac{\left(\delta+\lambda_{k}\right)-c \frac{\partial \cdot}{\partial u}-(c-a) \frac{\partial \cdot}{\partial b}}{\lambda_{k}}\right) \frac{\partial W(u, b)}{\partial u}\right|_{u=b}, j=1, \ldots, n .
\end{aligned}
$$

\subsubsection{Erlang(2) interarrivals and exponential claims}

In the special case of $\operatorname{Erlang}(2, \lambda)$ distributed interclaim times and $\operatorname{Exp}(\alpha)$ distributed claim amounts, the integro-differential equation (38) can (similarly to the previous sections) be transformed into the ordinary linear differential equation with constant coefficients

$$
\begin{aligned}
& a^{2} V^{\prime \prime \prime}(z)+\left(2(\delta+\lambda) a-\alpha a^{2}\right) V^{\prime \prime}(z) \\
& +\left((\delta+\lambda)^{2}-2 \alpha(\delta+\lambda) a\right) V^{\prime}(z)+\left(\alpha \lambda^{2}-\alpha(\delta+\lambda)^{2}\right) V(z)=0,
\end{aligned}
$$

with a solution of the form

$$
V(z)=\hat{A}_{1} e^{\hat{R}_{1} z}+\hat{A}_{2} e^{\hat{R}_{2} z}+\hat{A}_{3} e^{\hat{R}_{3} z},
$$

where $\left\{\hat{R}_{1}, \hat{R}_{2}, \hat{R}_{3}\right\}$ denote the roots of the polynomial $P_{1}(R)=a^{2} R^{3}+\left(2(\delta+\lambda) a-\alpha a^{2}\right) R^{2}+\left((\delta+\lambda)^{2}-2 \alpha(\delta+\lambda) a\right) R+\left(\alpha \lambda^{2}-\alpha(\delta+\lambda)^{2}\right)$. 
It is easy to see that $P_{1}(R)$ has three real roots, two of which are negative. The condition $\lim _{z \rightarrow \infty} V(z)=0$ implies that if $\hat{R}_{3}$ refers to the positive root, $\hat{A}_{3}=0$.

Under the assumption of exponential claim amounts, the boundary conditions (39) can be rewritten as

$$
V^{\prime}(0)=-1 \quad \text { and } \quad V^{\prime \prime}(0)=\frac{\delta}{a} .
$$

Altogether this leads to the explicit solution

$$
V(z)=\hat{A}_{1} e^{\hat{R}_{1} z}+\hat{A}_{2} e^{\hat{R}_{2} z}
$$

with

$$
\hat{A}_{1}=\frac{\hat{R}_{2}+\frac{\delta}{a}}{\hat{R}_{1} \hat{R}_{2}-\hat{R}_{1}^{2}} \text { and } \hat{A}_{2}=\frac{\hat{R}_{1}+\frac{\delta}{a}}{\hat{R}_{2}^{2}-\hat{R}_{1} \hat{R}_{2}} \text {. }
$$

The function $V(z)$ is differentiable and bounded. Thus it fulfills the conditions of Rolski et al. [27, Thm.11.2.3] and is indeed the solution to the problem.

For $W(u, b)$, one has to solve

$$
\left(\delta+\lambda-c \frac{\partial \cdot}{\partial u}-(c-a) \frac{\partial \cdot}{\partial b}\right)^{2} W(u, b)=\lambda^{2} \int_{0}^{u} W(u-v, b) \alpha e^{-\alpha v} d v,
$$

which by applying the operator $\left(\frac{\partial \cdot}{\partial u}+\alpha\right)$ leads to the PDE

$$
\begin{aligned}
(\delta+\lambda-c & \left.\frac{\partial \cdot}{\partial u}-(c-a) \frac{\partial \cdot}{\partial b}\right)^{2} \frac{\partial W(u, b)}{\partial u} \\
& +\alpha\left(\delta+\lambda-c \frac{\partial \cdot}{\partial u}-(c-a) \frac{\partial \cdot}{\partial b}\right)^{2} W(u, b)-\alpha \lambda^{2} W(u, b)=0 .
\end{aligned}
$$

The boundary conditions simplify to

$$
\begin{gathered}
\lim _{b \rightarrow \infty} W(u, b)=0, \\
\left.\frac{\partial W(u, b)}{\partial u}\right|_{u=b}=1, \\
\left.c \frac{\partial^{2} W(u, b)}{\partial u^{2}}\right|_{u=b}+\left.(c-a) \frac{\partial^{2} W(u, b)}{\partial u \partial b}\right|_{u=b}=\delta, \\
\lim _{u \rightarrow \infty} W(u, u+z)=V(z),
\end{gathered}
$$

where $V(z)$ is given by (40). 
(42) is a homogeneous differential equation of third order with constant coefficients and we will construct a solution of the form

$$
W(u, b)=\sum_{k=0}^{\infty} e^{S^{(k)} b}\left(A_{1}^{(k)} e^{R_{1}^{(k)} u}+A_{2}^{(k)} e^{R_{2}^{(k)} u}+A_{3}^{(k)} e^{R_{3}^{(k)} u}\right) .
$$

where $R_{i}^{(k)}\left(S^{(k)}\right),(i=1,2,3)$, denote the roots of the polynomial

$$
P\left(R, S^{(k)}\right)=R\left((\delta+\lambda)-c R-(c-a) S^{(k)}\right)^{2}+\alpha\left((\delta+\lambda)-c R-(c-a) S^{(k)}\right)^{2}-\alpha \lambda^{2}
$$

for a given value of $S^{(k)}$.

The main idea is again that each of the above summands solves (42) and the combination of such solutions is used to match all the necessary boundary conditions. Substitution of each term in the original integro-differential equation (41) gives

$$
\frac{A_{1}^{(k)}}{R_{1}^{(k)}+\alpha}+\frac{A_{2}^{(k)}}{R_{2}^{(k)}+\alpha}+\frac{A_{3}^{(k)}}{R_{3}^{(k)}+\alpha}=0 .
$$

As in the case of the survival probability, the choice $A_{3}^{(k)}=0$ for all $k \geq 0$ turns out to be feasible and hence

$$
A_{2}^{(k)}=-\frac{R_{2}^{(k)}+\alpha}{R_{1}^{(k)}+\alpha} A_{1}^{(k)} .
$$

So we actually look for a solution of the form

$$
W(u, b)=\sum_{k=0}^{\infty} A_{1}^{(k)} e^{S^{(k)} b}\left(e^{R_{1}^{(k)} u}-\frac{R_{2}^{(k)}+\alpha}{R_{1}^{(k)}+\alpha} e^{R_{2}^{(k)} u}\right) .
$$

Condition (43) is automatically satisfied as long as $S^{(k)}<0$ for $k \geq 0$. In view of (40) and (46), define for $k=0$

$$
\begin{array}{lll}
A_{1}^{(0)}:=\hat{A}_{1}, & S^{(0)}:=\hat{R}_{1} & R_{1}^{(0)}:=-\hat{R}_{1}, \\
A_{1}^{(1)}:=\hat{A}_{2}, & S^{(1)}:=\hat{R}_{2} & R_{1}^{(1)}:=-\hat{R}_{2} .
\end{array}
$$

At the same time this choice already determines the values of $R_{2}^{(0)}$ and $R_{2}^{(1)}$. Note also that the combination of $R_{1}^{(0)}$ and $R_{1}^{(1)}$ is possible, since $P(-R, R)=-P_{1}(R)$. By construction, $R_{1}^{(0)}$ and $R_{1}^{(1)}$ are positive and for $S^{(i)}=\hat{R}_{i}$ the polynomial $P(R$, $\left.S^{(i)}\right)$ also has a negative root, the value of which is assigned to $R_{2}^{(i)}(i=0,1)$. If both $S^{(0)}+R_{2}^{(0)}$ and $S^{(1)}+R_{2}^{(1)}$ and all remaining sums $S^{(k)}+R_{i}^{(k)}, k \geq 2, i \in\{1,2\}$, are negative, condition (46) will be fulfilled. 
Let us now turn the attention to (44). Inserting the above choice of the first terms, this condition reads

$$
\begin{aligned}
&-\hat{A}_{1} \hat{R}_{1}-\hat{A}_{2} \hat{R}_{2}-\hat{A}_{1} \frac{R_{2}^{(0)}+\alpha}{\alpha-\hat{R}_{1}} R_{2}^{(0)} e^{\left(R_{2}^{(0)}+\hat{R}_{1}\right) b}-\hat{A}_{2} \frac{R_{2}^{(1)}+\alpha}{\alpha-\hat{R}_{2}} R_{2}^{(1)} e^{\left(R_{2}^{(1)}+\hat{R}_{2}\right) b} \\
&+\sum_{k=2}^{\infty} A_{1}^{(k)}\left(R_{1}^{(k)} e^{\left(R_{1}^{(k)}+S^{(k)}\right) b}-\frac{R_{2}^{(k)}+\alpha}{R_{1}^{(k)}+\alpha} R_{2}^{(k)} e^{\left(R_{2}^{(k)}+S^{(k)}\right) b}\right)=1 .
\end{aligned}
$$

From $V^{\prime}(0)=-1$ we know that $\hat{A}_{1} \hat{R}_{1}+\hat{A}_{2} \hat{R}_{2}=-1$, so the above equation can be simplified to

$$
\begin{aligned}
-\hat{A}_{1} \frac{R_{2}^{(0)}+\alpha}{\alpha-\hat{R}_{1}} & R_{2}^{(0)} e^{\left(R_{2}^{(0)}+\hat{R}_{1}\right) b}-\hat{A}_{2} \frac{R_{2}^{(1)}+\alpha}{\alpha-\hat{R}_{2}} R_{2}^{(1)} e^{\left(R_{2}^{(1)}+\hat{R}_{2}\right) b} \\
& +\sum_{k=2}^{\infty} A_{1}^{(k)}\left(R_{1}^{(k)} e^{\left(R_{1}^{(k)}+S^{(k)}\right) b}-\frac{R_{2}^{(k)}+\alpha}{R_{1}^{(k)}+\alpha} R_{2}^{(k)} e^{\left(R_{2}^{(k)}+S^{(k)}\right) b}\right)=0,
\end{aligned}
$$

which will be fulfilled by an appropiate definition of the coefficients $A_{1}^{(k)}$. However, in addition we have to satisfy the boundary condition (45). Inserting all the chosen initial values and using the identity $\hat{A}_{1} \hat{R}_{1}^{2}+\hat{A}_{2} \hat{R}_{2}^{2}=\delta / a$, (45) can be written as

$$
\begin{aligned}
& -\hat{A}_{1} R_{2}^{(0)} \frac{R_{2}^{(0)}+\alpha}{\alpha-\hat{R}_{1}}\left(c R_{2}^{(0)}+(c-a) \hat{R}_{1}\right) e^{\left(R_{2}^{(0)}+\hat{R}_{1}\right) b} \\
& -\hat{A}_{2} R_{2}^{(1)} \frac{R_{2}^{(1)}+\alpha}{\alpha-\hat{R}_{2}}\left(c R_{2}^{(1)}+(c-a) \hat{R}_{2}\right) e^{\left(R_{2}^{(1)}+\hat{R}_{2}\right) b} \\
& +\sum_{k=2}^{\infty}\left(A_{1}^{(k)} R_{1}^{(k)}\left(c R_{1}^{(k)}+(c-a) S^{(k)}\right) e^{\left(R_{1}^{(k)}+S^{(k)}\right) b}\right. \\
& \left.\quad-A_{1}^{(k)} R_{2}^{(k)} \frac{R_{2}^{(k)}+\alpha}{R_{1}^{(k)}+\alpha}\left(c R_{2}^{(k)}+(c-a) S^{(k)}\right) e^{\left(R_{2}^{(k)}+S^{(k)}\right) b}\right)=0
\end{aligned}
$$

The procedure needed now to create the correction terms for these two remaining boundary conditions is analogous to the case of the survival probability in Section 3.1.1 and will therefore not be given in detail. The additional factor $\delta$ in all the polynomial equations does not cause any harm. A different feature of the present case as compared to Section 3.1.1 is that in order to satisfy condition (46), here coefficients for both the steps $k=0$ and $k=1$ have to be assigned, so we start with two (instead of one) terms to be deleted and the algorithm of Section 3.1.1 has to be applied to each of the two separately; the sequence $\left(S^{(k)}\right)_{k \in \mathbb{N}}$ then consists of strictly decreasing subsequences and tends to $-\infty$ again. 


\subsubsection{Numerical Illustration}

In the following, the exact solutions derived in the previous sections are approximated by truncating the series after 18 terms. The accuracy of this approximation is already striking. One should note that these values are obtained virtually instantaneously, whereas Monte Carlo simulation (including variance reduction procedures) takes several minutes to achieve a comparable accuracy. Tables 1 and 2 show exact and simulated values of the survival probability $U^{\text {lin }}(u, b)$ and

TABLE 1

EXACT AND SIMULATED VALUES FOR THE SURVIVAL PROBABILITY AND THE EXPECTED SUM OF DISCOUNTED DIVIDEND PAYMENTS FOR $\lambda=n=2, \alpha=1, \delta=0.03, c=1.5, a^{l i n}=0.8$.

\begin{tabular}{c|c|c|c|c}
\hline \hline & \multicolumn{2}{|c|}{$U^{\text {lin }}(u, 3)$} & \multicolumn{2}{c}{$W^{\text {lin }}(u, 3)$} \\
\hline$u$ & Exact & Simulation & Exact & Simulation \\
\hline 2.1 & 0.733224 & 0.7380 & 1.46862 & 1.46972 \\
2.2 & 0.739212 & 0.7333 & 1.54505 & 1.53293 \\
2.3 & 0.744364 & 0.7462 & 1.62477 & 1.65721 \\
2.4 & 0.748668 & 0.7506 & 1.70782 & 1.72777 \\
2.5 & 0.752118 & 0.7574 & 1.79422 & 1.80451 \\
2.6 & 0.754721 & 0.7543 & 1.88392 & 1.89729 \\
2.7 & 0.756511 & 0.7542 & 1.97677 & 1.95437 \\
2.8 & 0.757559 & 0.7615 & 2.07247 & 2.04798 \\
2.9 & 0.758001 & 0.7540 & 2.17051 & 2.18002 \\
3.0 & 0.758073 & 0.7554 & 2.27010 & 2.27564 \\
\hline \hline
\end{tabular}

TABLE 2

EXACT AND SIMULATED VALUES FOR THE SURVIVAL PROBABILITY AND THE EXPECTED SUM OF DISCOUNTED DIVIDEND PAYMENTS FOR $\lambda=4, n=2, \alpha=1.5, \delta=0.03, c=5 / 3, a^{l i n}=1 / 3$.

\begin{tabular}{c|c|c|c|c}
\hline \hline & \multicolumn{2}{|c|}{$U^{\text {lin }}(u, 2)$} & \multicolumn{2}{c}{$W^{\text {lin }}(u, 2)$} \\
\hline$u$ & Exact & Simulation & Exact & Simulation \\
\hline 1.1 & 0.518345 & 0.5226 & 0.000442681 & 0.00060890 \\
1.2 & 0.536764 & 0.5355 & 0.000897554 & 0.00112367 \\
1.3 & 0.554457 & 0.5453 & 0.001819520 & 0.00169736 \\
1.4 & 0.571422 & 0.5630 & 0.003687090 & 0.00398161 \\
1.5 & 0.587612 & 0.5858 & 0.007464610 & 0.00782931 \\
1.6 & 0.602876 & 0.6070 & 0.015079200 & 0.01474820 \\
1.7 & 0.616821 & 0.6167 & 0.030302600 & 0.02912370 \\
1.8 & 0.628532 & 0.6319 & 0.060134400 & 0.06046390 \\
1.9 & 0.636225 & 0.6347 & 0.115667000 & 0.11660100 \\
2.0 & 0.638223 & 0.6319 & 0.204578000 & 0.20514300 \\
\hline \hline
\end{tabular}


TABLE 3

COMPARISON FOR $\alpha=2, \lambda=2, \delta=0.03, c=1.1, a^{l i n}=0.55, a^{\text {thr }}=0.55$.

\begin{tabular}{c|c|c|c|c|c|c}
\hline \hline$u$ & $U^{\text {lin }}(u, 2)$ & $U^{\text {thr }}(u, 35)$ & $W^{\text {lin }}(u, 2)$ & $W^{\text {thr }}(u, 35)$ & $U^{\text {thr }}(u, 15)$ & $W^{\text {thr }}(u, 15)$ \\
\hline 1.0 & 0.910725 & 0.912509 & 2.47362 & 2.94955 & 0.912509 & 7.89945 \\
1.1 & 0.921141 & 0.923443 & 2.56674 & 2.99669 & 0.923443 & 8.02571 \\
1.2 & 0.930043 & 0.933011 & 2.66011 & 3.03995 & 0.933011 & 8.14155 \\
1.3 & 0.937560 & 0.941383 & 2.75400 & 3.07981 & 0.941383 & 8.24831 \\
1.4 & 0.943794 & 0.948709 & 2.84862 & 3.11672 & 0.948709 & 8.34716 \\
1.5 & 0.948818 & 0.955119 & 2.94414 & 3.15106 & 0.955119 & 8.43914 \\
1.6 & 0.952685 & 0.960728 & 3.04064 & 3.18318 & 0.960728 & 8.52515 \\
1.7 & 0.955437 & 0.965636 & 3.13812 & 3.21335 & 0.965636 & 8.60597 \\
1.8 & 0.957132 & 0.969931 & 3.23650 & 3.24185 & 0.969931 & 8.68228 \\
1.9 & 0.957896 & 0.973689 & 3.33565 & 3.26888 & 0.973689 & 8.75468 \\
2.0 & 0.958029 & 0.976977 & 3.43538 & 3.29465 & 0.976977 & 8.82371 \\
\hline \hline
\end{tabular}

the expected discounted dividends $W^{l i n}(u, b)$ in the linear barrier model with Erlang(2) interclaim times and exponential claim sizes for two different parameter sets. In these tables, the Monte Carlo estimates are based on $N=10000$ iterations. As an illustration, for $N=20000$ iterations, one obtains the simulation estimates $U^{l i n}(1.1,2)=0.51925$ and $W^{l i n}(1.1,2)=0.00046$ in Table 2, which is still not fully satisfying. One should note in this context that opposed to horizontal barrier models, here one has positive probability of survival of the trajectories, which increases the simulation time and effort.

\section{COMPARING THE TWO DIVIDEND MODELS}

The availability of exact solutions provides a quick way to compare the two dividend models investigated in this paper. One motivation for the introduction of the threshold dividend model was the positive survival probability, while the expected discounted dividend payments are still of reasonable size. Let us assume that we consider the survival probabilities $U$ and the expected sums of discounted dividend payments $W$ as the only quantities of interest and that we compare the two proposed dividend strategies at time 0 on that basis. Then, at least for larger values of the discounting factor, dividends earned at a rather late stage do not provide a substantial contribution to the overall sum of discounted dividend payments and hence it seems preferable to focus on securing survival once time has evolved. At the same time, dividends paid out at an earlier stage contribute significantly to the overall value of the discounted sum of dividend payments. For large $\delta$, this aspect is perhaps better captured by linear dividend barrier models (where the barrier departs from the ruin level as time evolves) than by threshold models (where the payment strategy is not "safer" at later times). 
The numerical values worked out in this section are intended to give an impression on how the performance of the dividend strategy differs in various regions of the parameter space.

In Table 3, for a given set of parameters including the value $b^{\text {lin }}$ of the linear barrier model, the threshold $b^{t h r}$ is calibrated in such a way that the expected dividend payments of the two strategies (which are calculated from (47), (17) and (18)) are of comparable size. It turns out that in this case also the survival probabilities (computed from (30), (20) and (22)) are comparable. However, if instead $b^{t h r}$ is more than halved, then the survival probabilities are not affected,

TABLE 4

COMPARISON FOR $\alpha=0.5, \lambda=4, c=4.2, \delta=0.08, a^{l i n}=3.6, a^{t h r}=0.1$.

\begin{tabular}{c|c|c|c|c}
\hline \hline$u$ & $U^{\text {lin }}(u, 20)$ & $U^{\text {thr }}(u, 25)$ & $W^{\text {lin }}(u, 20)$ & $W^{\text {thr }}(u, 25)$ \\
\hline 10 & 0.270068 & 0.221158 & 0.972399 & 0.147233 \\
11 & 0.285057 & 0.235981 & 1.171740 & 0.166242 \\
12 & 0.298725 & 0.250344 & 1.410030 & 0.187142 \\
13 & 0.311005 & 0.264260 & 1.695140 & 0.210175 \\
14 & 0.321820 & 0.277744 & 2.036520 & 0.235608 \\
15 & 0.331085 & 0.290809 & 2.445470 & 0.263735 \\
16 & 0.338698 & 0.303468 & 2.935500 & 0.294881 \\
17 & 0.344551 & 0.315733 & 3.522600 & 0.329407 \\
18 & 0.348533 & 0.327617 & 4.225038 & 0.367710 \\
19 & 0.350586 & 0.339132 & 5.059620 & 0.410232 \\
20 & 0.351000 & 0.350288 & 6.019980 & 0.457460 \\
\hline \hline
\end{tabular}

TABLE 5

COMPARISON FOR $\alpha=2, \lambda=2, c=0.8, a^{l i n}=0.6, a^{t h r}=0.25$.

\begin{tabular}{c|c|c|c|c|c|c}
\hline \hline & & & \multicolumn{2}{|c|}{$\delta=0.03$} & \multicolumn{2}{c}{$\delta=0.1$} \\
\hline$u$ & $U^{\text {lin }}(u, 1.5)$ & $U^{\text {thr }}(u,=2.5)$ & $W^{\text {lin }}(u, 1.5)$ & $W^{\text {thr }}(u,=2.5)$ & $W^{\text {lin }}(u, 1.5)$ & $W^{\text {thr }}(u,=2.5)$ \\
\hline 0.5 & 0.598238 & 0.522446 & 2.84655 & 4.13162 & 0.98854 & 0.919823 \\
0.6 & 0.619711 & 0.545412 & 2.99965 & 4.33540 & 1.06783 & 0.976708 \\
0.7 & 0.637969 & 0.566298 & 3.14423 & 4.52543 & 1.14843 & 1.032120 \\
0.8 & 0.653200 & 0.585293 & 3.28100 & 4.70307 & 1.23070 & 1.086340 \\
0.9 & 0.665577 & 0.602567 & 3.41055 & 4.86955 & 1.31493 & 1.139630 \\
1.0 & 0.675266 & 0.618277 & 3.53345 & 5.02600 & 1.40141 & 1.192220 \\
1.1 & 0.682442 & 0.632565 & 3.65021 & 5.17343 & 1.49034 & 1.244320 \\
1.2 & 0.687309 & 0.645560 & 3.76139 & 5.31275 & 1.58188 & 1.296120 \\
1.3 & 0.690138 & 0.657380 & 3.86771 & 5.44480 & 1.67609 & 1.347810 \\
1.4 & 0.691330 & 0.668132 & 3.97017 & 5.57032 & 1.77286 & 1.399530 \\
1.5 & 0.691525 & 0.677914 & 4.07045 & 5.69000 & 1.87191 & 1.451440 \\
\hline \hline
\end{tabular}


TABLE 6

COMPARISON FOR $\alpha=0.5, \lambda=2, c=2.5, a^{l i n}=2, a^{\text {thr }}=0.4$.

\begin{tabular}{c|c|c|c|c|c|c}
\hline \hline & \multicolumn{2}{|c|}{} & \multicolumn{2}{|c|}{$\delta=0.03$} & \multicolumn{2}{c}{$\delta=0.1$} \\
\hline$u$ & $U^{\text {lin }}(u, 10)$ & $U^{\text {thr }}(u, 20)$ & $W^{\text {lin }}(u, 10)$ & $W^{\text {thr }}(u, 20)$ & $W^{\text {lin }}(u, 10)$ & $W^{\text {thr }}(u, 20)$ \\
\hline 9.0 & 0.611476 & 0.622957 & 8.38890 & 5.44013 & 14.2771 & 22.0554 \\
9.1 & 0.611840 & 0.625355 & 8.48463 & 5.47752 & 14.3777 & 22.1631 \\
9.2 & 0.612144 & 0.627722 & 8.58098 & 5.51489 & 14.4781 & 22.2699 \\
9.3 & 0.612390 & 0.630058 & 8.67791 & 5.55226 & 14.5784 & 22.3761 \\
9.4 & 0.612584 & 0.632363 & 8.77540 & 5.58962 & 14.6786 & 22.4814 \\
9.5 & 0.612729 & 0.634639 & 8.87343 & 5.62698 & 14.7788 & 22.5860 \\
9.6 & 0.612831 & 0.636886 & 8.97194 & 5.66435 & 14.8788 & 22.6900 \\
9.7 & 0.612895 & 0.639104 & 9.07090 & 5.70172 & 14.9789 & 22.7931 \\
9.8 & 0.612930 & 0.641292 & 9.17025 & 5.73910 & 15.0788 & 22.8956 \\
9.9 & 0.612944 & 0.643453 & 9.26991 & 5.77649 & 15.1788 & 22.9974 \\
10.0 & 0.612946 & 0.645586 & 9.36982 & 5.81389 & 15.2788 & 23.0986 \\
\hline \hline
\end{tabular}

TABLE 7

COMPARISON FOR $\alpha=0.25, \lambda=2, c=4.2, \delta=0.02, a^{l i n}=2.3, a^{t h r}=0.19$.

\begin{tabular}{c|c|c|c|c}
\hline \hline$u$ & $U^{l i n}(u, 15)$ & $U^{\text {thr }}(u, 20)$ & $W^{l i n}(u, 15)$ & $W^{\text {thr }}(u, 20)$ \\
\hline 14.0 & 0.210288 & 0.00589161 & 3.36797 & 3.50556 \\
14.1 & 0.210418 & 0.00599345 & 3.45753 & 3.52634 \\
14.2 & 0.210526 & 0.00609513 & 3.54874 & 3.54716 \\
14.3 & 0.210615 & 0.00619665 & 3.64152 & 3.56801 \\
14.4 & 0.210685 & 0.00629801 & 3.73579 & 3.58889 \\
14.5 & 0.210737 & 0.00639921 & 3.83143 & 3.60981 \\
14.6 & 0.210774 & 0.00650025 & 3.92832 & 3.63076 \\
14.7 & 0.210797 & 0.00660113 & 4.02628 & 3.65174 \\
14.8 & 0.210809 & 0.00670185 & 4.12513 & 3.67275 \\
14.9 & 0.210814 & 0.00680241 & 4.22464 & 3.69389 \\
15.0 & 0.210815 & 0.00690281 & 4.32454 & 3.71488 \\
\hline \hline
\end{tabular}

whereas the expected dividends are much higher (see the two columns on the right of Table 3).

Table 4 depicts a situation where $a^{t h r}$ and $b^{t h r}$ are chosen so that the ruin probabilities of the two strategies are comparable, but where then the linear barrier strategy outperforms the layer strategy in terms of expected dividend payments. Table 5 illustrates the importance of the discount factor $\delta$ in comparing the performance of the two strategies. Table 6 shows a parameter choice with comparable survival probabilities, where the linear barrier strategy is preferable although the discount factor is of moderate size $(\delta=0.03)$. For still smaller 
values of $\delta$, the situation is reversed again (see the two columns on the right of Table 6).

This indicates that for every parameter setting and initial surplus $u$ a critical value $\delta^{*}(u)$ exists such that for $\delta>\delta^{*}(u)$ the linear barrier strategy performs better and for $\delta<\delta^{*}(u)$ the threshold strategy is to be preferred (while the level of survival probability is not affected by the choice of $\delta$ ).

Finally, in Table 7 we indicate a combination of parameters, for which much more risk must be taken with the threshold strategy to achieve expected dividends of the order of the linear barrier model.

\section{CONCLUSION}

The problem of determining the optimal dividend strategy that maximizes the expected discounted dividend payments until ruin and at the same time does not lead to ruin with a specified probability bound is currently out of sight. An alternative approach is to formulate explicit strategies and to tune the involved parameters in such a way that a given target in terms of profitability and solvency is reached. For that approach explicit expressions in terms of the model parameters are necessary. In this paper we showed how to obtain such expressions for the Erlang renewal model. Using these explicit expressions, a comparison of a barrier strategy that evolves linearly in time with a threshold strategy (which depends on the surplus level) showed that the former can outperform the latter in terms of the usual optimization criteria (namely expected sum of discounted dividend payments versus survival probability). This shows that although a strategy that depends on time is perhaps less intuitive than one depending on the surplus level, it may be preferable in some situations, although most of the research literature is focussed on surplus-dependent strategies. In particular the results of this paper show that one has to be very careful about the choice of the dividend payout strategy.

Moreover, it should be kept in mind that even when ignoring the safety aspect and purely looking at expected profits, the higher moments of the discounted dividend payments under horizontal and threshold strategies in the literature can be considerably large (see for instance Albrecher et al. [1]), making the sole use of this optimization criterion somewhat questionable. One other way to improve upon that might be a utility-based approach. However, identifying corresponding optimal dividend strategies is in general extremely difficult; for recent progress in a diffusion framework see Hubalek \& Schachermayer [22] and Gerber \& Shiu [18].

\section{REFERENCES}

[1] Albrecher, H., Claramunt, M.M. and Mármol, M. (2005) On the distribution of dividend payments in a Sparre Andersen model with generalized $\operatorname{Erlang}(n)$ interclaim times. Insurance: Mathematics \& Economics, 37(2), 324-334. 
[2] Albrecher, H. and Hartinger, J. (2006) On the non-optimality of horizontal dividend barrier strategies in the Sparre Andersen model. Hermis J. Comp. Math. Appl., 7, 1-14.

[3] Albrecher, H., Hartinger, J. and Tichy, R.F. (2005) On the distribution of dividend payments and the discounted penalty function in a risk model with linear dividend barrier. Scand. Actuar. J., 2, 103-126.

[4] Albrecher, H. and Kainhofer, R. (2002) Risk theory with a non-linear dividend barrier. Computing, 68(4), 289-311.

[5] Asmussen, S. (2000) Ruin probabilities. World Scientific, Singapore.

[6] Azcue, P. and Muler, N. (2005) Optimal reinsurance and dividend distribution policies in the Cramér-Lundberg model. Math. Finance, 15(2), 261-308.

[7] Cheng, Y. and Tang, Q. (2003) Moments of the surplus before ruin and the deficit at ruin in the Erlang(2) risk process. North American Actuarial Journal 7, 1-12.

[8] Cohen, J. and Down, D. (1996) On the role of Rouché's theorem in queueing analysis. Queueing Systems, 23, 281-291.

[9] DAvis, M.H.A. (1984) Piecewise-deterministic Markov processes: a general class of nondiffusion stochastic models. J. Roy. Statist. Soc. Ser. B, 46(3), 353-388.

[10] Dickson, D.C.M. (1998) On a class of renewal risk process. North American Actuarial Journal, 2(3), 60-73.

[11] Dickson, D.C.M. (2005) Insurance Risk and Ruin. Cambridge University Press.

[12] Dickson, D.C.M. and HipP, C. (1998) Ruin probabilities for Erlang(2) risk process. Insurance Math. Econom., 22, 251-262.

[13] Dickson, D.C.M. and HipP, C. (2001) On the time to ruin for Erlang(2) risk process. Insurance Math. Econom., 29, 333-344.

[14] Dickson, D. and Waters, H. (2004) Some optimal dividend problems. Astin Bulletin, 34(1), 49-74.

[15] Gerber, H.U. (1969) Entscheidungskriterien fuer den zusammengesetzten Poisson-Prozess. Schweiz. Aktuarver. Mitt., (1), 185-227.

[16] Gerber, H.U. (1979) An Introduction to Mathematical Risk Theory. Huebner Foundation Monograph 8, Homewood, Ill.

[17] Gerber, H.U. (1981) On the probability of ruin in the presence of a linear dividend barrier. Scand. Actuar. J., (1), 105-115.

[18] Gerber, H.U. and Shiu, E. (2004) Optimal dividends: Analysis with Brownian motion. North American Actuarial Journal, 8(1), 1-20.

[19] Gerber, H.U. and Shiu, E. (2005) The time value of ruin in a Sparre Andersen model. North American Actuarial Journal, 9(2), 49-84.

[20] Gerber, H.U. and Shiu, E. (2006) On optimal dividends: from reflection to refraction. J. Comput. Appl. Math., 126, 4-22.

[21] Gerber, H.U. and Shiu, E. (2006) On optimal dividend strategies in the compound Poisson model. North American Actuarial Journal (to appear).

[22] Hubalek, F. and Schachermayer, W. (2004) Optimizing expected utility of dividend payments for a Brownian risk process and a peculiar nonlinear ODE. Insurance Math. Econom., 34, 193-225.

[23] Li, S. and Garrido, J. (2004) On ruin for the Erlang(n) risk process. Insurance Math. Econom., 34(3), 391-408.

[24] Li, S. and GARRIDO, J. (2004) A class of renewal risk models with a constant dividend barrier. Insurance Math. Econom., 35(3), 691-701.

[25] Lin, X. and Pavlova, K. (2006) The compound poisson risk model with a threshold dividend strategy. Insurance Math. Econom. (to appear).

[26] Lin, X.S., Willmot, G.E. and Drekic, S. (2003) The classical risk model with a constant dividend barrier: analysis of the Gerber-Shiu discounted penalty function. Insurance Math. Econom., 33(3), 551-566.

[27] Rolski, T., Schmidli, H., Schmidt, V. and Teugels, J. (1999) Stochastic processes for insurance and finance. Wiley Series in Probability and Statistics. John Wiley \& Sons Ltd., Chichester.

[28] Schmidui, H. (2006) Optimal control in insurance. Springer, Berlin (to appear).

[29] Siegl, T. and Tichy, R. (1996) Lösungsmethoden eines Risikomodells bei exponentiell fallender Schadensverteilung. Schweiz. Aktuarver. Mitt., (1), 85-118. 
[30] Sparre Andersen, E. (1957) On the collective theory of risk in the case of contagion between the claims. Transactions XVth Int. Congress of Actuaries, New York, (II), 219-229.

HANSJÖRG ALBRECHER

Graz University of Technology

Steyrergasse 30, A-8010 Graz

Austria

and

Radon Institute for Computational and Applied Mathematics

Austrian Academy of Sciences

Altenbergerstrasse 69, A-4040 Linz

Austria

JÜRGEN HARTINGER

Radon Institute for Computational and Applied Mathematics

Austrian Academy of Sciences

Altenbergerstrasse 69, A-4040 Linz

Austria

Stefan Thonhauser

Radon Institute for Computational and Applied Mathematics

Austrian Academy of Sciences

Altenbergerstrasse 69, A-4040 Linz

Austria 